\title{
CHINESE UNKNOWN WORD IENTIFICATION AS KNOWN WORD TAGGING
}

\author{
GUO-HONG FU, KANG-KWONG LUKE \\ Department of Linguistics, The University of Hong Kong, Pokfulam Road, Hong Kong \\ E-MAIL: ghfu@hkucc.hku.hk, kkluke@hkuusa.hku.hk
}

\begin{abstract}
:
This paper presents a tagging approach to Chinese unknown word identification based on lexicalized hidden Markov models (LHMMs). In this work, Chinese unknown word identification is represented as a tagging task on a sequence of known words by introducing word-formation patterns and part-of-speech. Based on the lexicalized HMMs, a statistical tagger is further developed to assign each known word an appropriate tag that indicates its pattern in forming a word and the part-of-speech of the formed word. The experimental results on the Peking University corpus indicate that the use of lexicalization technique and the introduction of part-of-speech are helpful to unknown word identification. The experiment on the SIGHAN-PK open test data also shows that our system can achieve state-of-art performance.
\end{abstract}

Keywords:

Unknown word identification; lexicalized HMMs; known word tagging; Chinese word segmentation

\section{Introduction}

Unknown word identification (UWI) is an important and difficult problem in Chinese word segmentation. On the one hand, most current systems for Chinese word segmentation are based on a predefined machine-readable dictionary. However, no dictionary can be complete. In general, some $8-10 \%$ of words in real text are out of the dictionaries in use. Therefore, a practical system for Chinese word segmentation must be capable of detecting these out-of-vocabulary or unknown words. On the other hand, Chinese UWI is by no means a trivial task in that Chinese unknown words are constructed dynamically and freely. In theory, any combination of Chinese characters or lexicon words may be a potential unknown word. However, there lack of enough explicit marks in plain Chinese texts, such as capitalization in English that can be used directly to identify unknown words. Consequently, the exploration of more potential features is usually an effective way to improve the systems for Chinese UWI.

In the past years, a variety of techniques have been proposed to address the problem of Chinese UWI. Each technique has its own deficiencies while offering its advantages. Wu and Jiang took word segmentation and UWI as an integral part of full sentence analysis [1]. This method is proved to be powerful in segmentation disambiguation and UWI. However, the coverage of the parser may restrict its applications in practical NLP systems. Zhang et al presented a novel method to Chinese UWI based on role tagging [2]. They defined a set of unknown word roles about varied internal components and contexts. As a result, their system can detect different types of unknown words in real text. However, an additional role-tagged corpus is needed to learn role knowledge, which is not always available in practice. Xue recently reported a supervised machine-learning approach to Chinese word segmentation [3]. In his work, Chinese word segmentation is re-formulated as a problem of tagging Chinese character with position-of-character (POC) tags. This approach does not need a dictionary at all, so it is effective in principle for UWI. However, this method is purely based on character tagging, which may lose the important word-level features for correct disambiguation and UWI. More recently, Fu and Luke proposed a modified class-based LM approach to Chinese UWI [4]. In their work, Chinese UWI is viewed as a classification problem, and a number of different features, including contextual class feature, word juncture model and word formation patterns, are combined in a class-based LM framework to identify different unknown words. However, it is still an open problem to normalize different probabilistic distributions of different dimensions in an optimal way.

In this paper, we propose a lexicalized hidden Markov model (LHMM) approach to Chinese UWI. In this work, Chinese UWI is represented as a tagging task on a sequence of known words by introducing word-formation patterns. To do this, a tagger is thus developed based on the lexicalized HMMs to assign each known word in input an appropriate tag that indicates its patterns in forming a word and the part-of-speech of this formed word. In comparison with standard HMMs, the lexicalized HMMs can handle 
richer contextual information, both contextual words and tags for correct tagging of known, words. In addition, part-of-speech tags are also introduced and incorporated with the word-formation pattern tags. In this way, most Chinese unknown words can be resolved effectively.

The rest of this paper is organized as follows: Section 2 discusses how Chinese UWI can be reformulated as known word tagging. Section 3 presents the lexicalized HMMs for unknown word tagging. In section 4, the tagging algorithm is given in brief. Finally, the experimental results and some conclusions on this work will be given respectively in section 5 and section 6 .

\section{Chinese UWI as known word tagging}

In this section, Chinese UWI is represented as known word tagging by introducing word-formation pattern and part-of-speech tags.

\subsection{Representing segmented words with pattern-tags}

In practice, known words and unknown words in a sentence can be represented by means of word-formation pattern tags. As discussed in [4], a lexicon word $w$ has four possible word-formation patterns to present itself after UWI: (1) $w$ is an independent segmented known word by itself; (2) $w$ is at the beginning of an unknown word. (3) $w$ is at the middle of an unknown word. (4) $w$ is at the end of an unknown word. For convenience, these patterns are denoted respectively by four tags, i.e. $I S W, B O W, M O W$ and $E O W$.

Obviously, an unknown word will be resolved once the relevant word-formation patterns of its components are determined. At this point, Chinese UWI is equivalent to a process of assigning of word-formation pattern tags on a sequence of known words. More formally, a lexicon word may be tagged with four possible tags shown above in terms of its patterns during UWI: It will be tagged as $I S W$ if it is recognized as an independent known word during UWI; On the contrary, it will be tagged as $B O W, M O W$ or $E O W$ respectively if it present itself at the beginning, middle or end of an unknown word after UWI.

For example, the segmented sentence “中国/国家/主 席/胡/锦涛/同/北朝鲜/领导人/金/正日/举行/会谈/。 (Chinese President Hu Jintao held talks with North Korean leader Kim Jong-Il) can be represented using the pattern tags as follows:

$<\mathrm{ISW}>$ 中国 $<\mathrm{ISW}><\mathrm{ISW}>$ 国家 $<\mathrm{ISW}><\mathrm{ISW}>$ 主席 $<$ ISW $><$ ISW $>$ 胡 $<$ ISW $><$ BOW $>$ 锦 $<$ BOW $><$ EOW $>$ 涛 $<$ EOW $><$ ISW $>$ 同 $<$ ISW $><$ ISW $>$ 北朝鲜 $</$ ISW $><$ ISW $>$ 领导人 $<\mathrm{ISW}><\mathrm{ISW}>$ 金 $<\mathrm{ISW}><\mathrm{BOW}>$ 正 $<\mathrm{BOW}>$
$<$ EOW $>$ 日 $<$ EOW $><$ ISW $>$ 举行 $<$ ISW $><$ ISW $>$ 会谈 $<$ ISW $><$ ISW $>$ 。 $<$ ISW $>$

Differing from Xue's formulation [3], our formulation is based on known word tagging, which has two main advantages: Firstly, the word-based formulation is more general in that any unknown word must be made up of a number of known words, including single-character or multi-character known words. The second advantage of the formulation based on known word tagging is that it allows the use of more important word-level information such as contextual words and tags for ambiguity resolution and UWI.

\subsection{Incorporating POS-tag with pattern-tag for UWI}

It has been proved that part-of-speech is another important information for correct UWI [1][4], part-of-speech tags are accordingly introduced in this work. For convenience, we merge part-of-speech tags and the pattern tags by using following format: T1-T2. Where T1 denotes a part-of-speech tag and T2 denotes a word-formation pattern tag. Note that the Peking University part-of-speech tag-set is used in our system, which contains 48 different tags. With this combined tag-set, the previous example can be further represented as follows:

$<\mathrm{ns}-\mathrm{ISW}>$ 中国 $<\mathrm{ns}-\mathrm{ISW}><\mathrm{n}-\mathrm{ISW}>$ 国家 $<\mathrm{n}$-ISW $>$ $<$ n-ISW $>$ 主席 $<$ nn-ISW $><$ nr-ISW $>$ 胡 $<$ nr-ISW $><$ nr-BOW $>$ 锦 $</$ nr-BOW $><$ nr-EOW $>$ 涛 $</ n r-E O W\rangle<$-ISW $>$ 同 $<$ p-ISW $><$ ns-ISW $>$ 北朝鲜 $<$ ns - ISW $><n-I S W>$ 领导人 $<$ n-ISW $><$ nr-ISW $>$ 金 $<$ nnr-ISW $><$ nr-BOW $>$ 正 $</$ nr-BO W $><$ nr-EOW $>$ 日 $<$ nr-EOW $><$ v-ISW $>$ 举行 $</ v-I S W>$ $<$ vn -ISW $>$ 会谈 $</$ vn-ISW $><$ W-ISW $>$ 。 $<$ w-ISW $>$

\section{Lexicalized HMMs for Chinese UWI}

The lexicalized HMM approach has been widely used in POS tagging [5], shallow parsing [6] and Chinese prosodic phrase prediction [7]. In this section, we continue to apply it to perform the known tagging for Chinese UWI.

\subsection{Lexicalized HMMs}

From the statistical point of view, the task of known word tagging for Chinese UWI can be defined as the process of finding an appropriate tag sequence $\hat{T}=t_{1} t_{2} \cdots t_{n}$ that maximizes the conditional probability $P(T \mid W)$, given a sequence of known words $W=w_{1} w_{2} \cdots w_{n}$, namely,

$$
\hat{T}=\underset{T}{\arg \max } P(T \mid W)=\underset{T}{\arg \max } \frac{P(W \mid T) P(T)}{P(W)}
$$


Proceedings of the Third International Conference on Machine Learning and Cybernetics, Shanghai, 26-29 August 2004

For a specific sequence of known words $w$, the probability $P(W)$ is fixed. Therefore, it can be dropped from the above equation. Thus, we have a general statistical model for known word tagging as follows:

$$
\begin{aligned}
& \hat{T}=\underset{T}{\arg \max } P(W \mid T) P(T) \\
& =\underset{T}{\arg \max } P\left(w_{1, n}, t_{1, n}\right) \\
& =\underset{T}{\arg \max } \prod_{i=1}^{n} P\left(w_{i} \mid w_{1, i-1}, t_{1, i}\right) P\left(t_{i} \mid w_{1, i-1}, t_{1, i-1}\right)
\end{aligned}
$$

However, this general model is not computable in practice because it has too many parameters. To address this problem, two types of approximations are employed here to make it applicable.

The first approximation is based on the independent hypothesis in standard HMMs: The appearance of current word $w_{i}$ depends only on current tag $t_{i}$ during known word tagging, and the assignment of current tag $t_{i}$ depends only on its previous tag $t_{i-1}$. Thus,

$$
\hat{T}=\underset{T}{\arg \max } \prod_{i=1}^{n} P\left(w_{i} \mid t_{i}\right) P\left(t_{i} \mid t_{i-1}\right)
$$

Equation (3) actually presents a first-order HMMs for known word tagging. Where, $P\left(w_{i} \mid t_{i}\right)$ is the so-called lexical probability; and $P\left(t_{i} \mid t_{i-1}\right)$ denotes the contextual tag probability.

The second type of approximation follows the notion of the lexicalized HMMs. In this approximation, the appearance of current word $w_{i}$ is assumed to depend not only on current tag $t_{i}$ but also its previous word $w_{i-1}$, and the assignment of current tag $t_{i}$ is supposed to depend both its previous word $w_{i-1}$ and its previous tag $t_{i-1}$. Thus, we have the lexicalized HMMs for UWI as follows:

$$
\hat{T}=\underset{T}{\arg \max } \prod_{i=1}^{n} P\left(w_{i} \mid w_{i-1}, t_{i}\right) P\left(t_{i} \mid w_{i-1}, t_{i-1}\right)
$$

In comparison with the standard HMMs, the lexicalized HMMs can provide richer contextual information for the assigning of tags to known words, including both contextual words and contextual tags, which will result in improvement of accuracy in UWI.

\subsection{Parameter estimation and data smoothing}

For simplification, we apply the maximum likelihood estimation (MLE) to estimate the parameters in Equation (3) and Equation (4). In MLE, parameters are estimated with their relative frequencies that are extracted directly from the manual corpus for training. The MLE of HMMs and LHMMs is formulated respectively in equation (5) and (6).

$$
\begin{aligned}
& \left\{\begin{array}{l}
P\left(w_{i} \mid t_{i}\right)=\frac{\operatorname{Count}\left(w_{i}, t_{i}\right)}{\operatorname{Count}\left(t_{i}\right)} \\
P\left(t_{i} \mid t_{i-1}\right)=\frac{\operatorname{Count}\left(t_{i-1}, t_{i}\right)}{\operatorname{Count}\left(t_{i-1}\right)}
\end{array}\right. \\
& \left\{\begin{array}{l}
P\left(w_{i} \mid w_{i-1}, t_{i}\right)=\frac{\operatorname{Count}\left(w_{i-1}, w_{i}, t_{i}\right)}{\operatorname{Count}\left(w_{i-1}, t_{i}\right)} \\
P\left(t_{i} \mid w_{i-1}, t_{i-1}\right)=\frac{\operatorname{Count}\left(w_{i-1}, t_{i-1}, t_{i}\right)}{\operatorname{Count}\left(w_{i-1}, t_{i-1}\right)}
\end{array}\right.
\end{aligned}
$$

Though the MLE has the advantage of simpleness, it will yield zero probabilities for any cases that are not observed in the training data. In our implementation, we employ the linear interpolation smoothing technique to avoid this problem of data sparseness. As shown equation (7), higher-order parameters in HMMs are smoothed with the relevant lower-order probabilities.

$$
\left\{\begin{array}{c}
P^{\prime}\left(w_{i} \mid t_{i}\right)=\lambda P\left(w_{i} \mid t_{i}\right)+\frac{1-\lambda}{\operatorname{Count}\left(t_{i}\right)} \\
P^{\prime}\left(t_{i} \mid t_{i-1}\right)=\mu P\left(t_{i} \mid t_{i-1}\right)+(1-\mu) P\left(t_{i}\right)
\end{array}\right.
$$

In smoothing the lexicalized HMMs, we use non-lexicalized probabilities to smooth the relevant lexicalized probabilities. This process is given in detail in equation (8).

$$
\left\{\begin{array}{c}
P^{\prime}\left(w_{i} \mid w_{i-1}, t_{i}\right)=\lambda P\left(w_{i} \mid w_{i-1}, t_{i}\right)+(1-\lambda) P\left(w_{i} \mid t_{i}\right) \\
P^{\prime}\left(t_{i} \mid w_{i-1}, t_{i-1}\right)=\mu P\left(t_{i} \mid w_{i-1}, t_{i-1}\right)+(1-\mu) P\left(t_{i} \mid t_{i-1}\right)
\end{array}\right.
$$

\section{The tagging algorithm}

Based on the models in equation (3) or (4), the tagging algorithm aims to score all possible candidate sequences of tags and find the best one that has the maximum score. In our system, this task is done by the classical Viterbi algorithm, which consists of two main steps: (1) The generation of candidate tags: The first step generates all possible candidate tags for each known word in the input by looking up the system dictionary or the library of lexical probabilities. All these candidate tags are stored in a lattice structure. (2) The decoding of best tags: In this step, the Viterbi algorithm scores all candidate tags with HMMs or LHMMs, and then searches the best path through the lattice built in the first step that maximizes the score. This path contains the best sequence of tags for the input sequence of known word sequence.

With this tagging algorithm, we develop a complete Chinese word segmenter using the two-stage strategy [4]. 
This system works in three main phrases, namely known word segmentation, tagging, and the conversion of known word tagged result to a sequence of segmented words. In order to yield correct segmentations for some complicated cases such as a mixture of ambiguities and unknown words in real texts, a pure known-word based n-gram is applied here to perform known word segmentation.

Similar to the work in [3], inconsistent tagging may occurs in our system. In practice, there are two types of inconsistent tagging in this work, namely the pattern inconsistency and the POS inconsistency. Pattern inconsistency arises when two adjacent known words are assigned inconsistent pattern tags such as "ISW : MOW" or "ISW : EOW". The part-of-speech inconsistency means that two adjacent known words are tagged with different part-of-speech while at the same time, they are assigned the pattern tags indicating they should occur in one unknown word. For example, the tag pair "a-BOW : n-EOW" is inconsistent in part-of-speech tagging. Since it has been proved that the inconsistent tagging hardly exerts any influence on the final results [3], we leave the inconsistent tagging as it is in our implementation. In fact, few inconsistent tags can occurs in the final result because they usually have lower probabilities, and will be mostly blocked by the decoder.

\section{Experiments}

In evaluating our approach, we conduct two experiments respectively on the Peking University corpus (January 1998 of the People's Daily) [8] and the PK-open test corpus for the First International Word Segmentation Bakeoff [9]. This section reports the relevant results of these experiments.

\subsection{Experimental data and evaluation measures}

In our experiments, we use the same corpora as used in [4], which come from two resources: The first one is from the Peking University corpus, which contains one month (January 1998) of news texts from the People's Daily, and has been manually segmented and tagged with part-of-speech by Peking University [8]. As shown in Table 1 , this corpus is separated into two parts: The larger part (viz. the Corpus $A$ ) is used to train our system, and the smaller part (viz. the Corpus B) is used for the closed-test. Furthermore, Corpus $A$ is automatically labeled with word-formation pattern tags by using the forward maximum matching technique. The second source (viz. the Corpus $($ ) is from SIGHAN bakeoff data, which is first used for the PK-open test at the First International Chinese Word Segmentation Bakeoff sponsored by SIGHAN [9], and is used here for the open comparison test.

Table 1. Experimental corpora

\begin{tabular}{|c|r|r|c|}
\hline Corpora & \# words & \#OOV words & OOV rate (\%) \\
\hline Corpus A & 998,085 & 68,638 & 6.88 \\
\hline Corpus B & 112,373 & 7,444 & 6.62 \\
\hline Corpus C & 17,605 & 1,619 & 9.20 \\
\hline
\end{tabular}

In addition to the above corpora, we also use a lexicon in our system, which is mainly built from the Peking University dictionary. In order to process the non-standard Chinese words in real texts, a number of non-Hanzi characters are also added in it. Consequently, the final dictionary contains about 65,270 different word-forms in all. Furthermore, all possible part-of-speech candidates of a word-form are also defined in it. Based on this lexicon, the relevant out-of-vocabulary rates (OOV rate for short) of the three corpora in Table 1 are $6.88 \%, 6.62 \%$ and $9.20 \%$ respectively.

In evaluating the effectiveness of our system, three measures are computed in our experiments, including recall $(\mathrm{R})$, precision $(\mathrm{P})$ and $F$-score $(\mathrm{F})$. Here, recall $(\mathrm{R})$ is defined as the number of correctly segmented words divided by the total number of words in the manually annotated corpus, and precision (P) is defined as the number of correctly segmented words divided by the total numbers of words segmented automatically by the system. As for F-score (denoted by $F$ ), it is the weighted harmonic mean of precision and recall that is formulated as follows:

$$
\text { - } \quad F=\frac{\left(\beta^{2}+1\right) \times P \times R}{\beta^{2} \times R+P}
$$

Here, we employ the balanced F-score (viz. $\beta^{2}=1$ ) to evaluate the overall performance of our system in word segmentation and UWI in that it is still not clear whether recall or precision is more important in evaluating a word segmentation system.

\subsection{Experimental results and discussions}

As mentioned above, the lexicalization technique and part-of-speech tags are introduced into the proposed approaches. The first experiment is therefore conducted to test how the introduction of the lexicalization technique or part-of-speech tags improves the performance of our system in word segmentation and UWI. The results are presented in Table 2. Each row in this table contains three lines of numbers, which denote the accuracy of the relevant approach respectively in word segmentation, known word segmentation and UWI. 
Proceedings of the Third International Conference on Machine Leaming and Cybernetics, Shanghai, 26-29 August 2004

Table 2. Experimental results on PKU corpus

\begin{tabular}{|l|c|c|c|}
\hline Methods & Recall(\%) & Precision(\%) & F-score(\%) \\
\hline HMMs & 94.65 & 93.12 & 93.88 \\
without & 97.57 & 94.13 & 95.82 \\
POS & 54.34 & 73.63 & 62.53 \\
\hline HMMs & 96.07 & 95.24 & 95.66 \\
With & 97.83 & 96.06 & 96.94 \\
POS & 71.83 & 82.11 & 76.63 \\
\hline LHMMs & 97.06 & 96.72 & 96.89 \\
without & 98.01 & 97.42 & 97.72 \\
POS & 84.05 & 86.54 & 85.28 \\
\hline LHMMs & $\mathbf{9 7 . 3 2}$ & $\mathbf{9 6 . 9 1}$ & $\mathbf{9 7 . 1 2}$ \\
With & $\mathbf{9 8 . 1 3}$ & $\mathbf{9 7 . 4 6}$ & $\mathbf{9 7 . 7 9}$ \\
POS & $\mathbf{8 6 . 2 0}$ & $\mathbf{8 9 . 0 3}$ & $\mathbf{8 7 . 5 9}$ \\
\hline
\end{tabular}

The data in Table 2 reveals two main findings. Firstly, the lexicalized HMMs perform better than the non-lexicalized HMMs. As can be seen in Table 2, the lexicalized HMMs improve the F-measure in UWI by 10.96 percents for the tag-set with part-of-speech and 23.75 percents for the tag-set without part-of-speech. Furthermore, the improvements of accuracy in UWI will contribute further 1.46 or 3.01 percents to the relevant overall F-score in word segmentation. Secondly, the introduction of part-of-speech tags is helpful to improve unknown word identification. It is shown in Table 2 that the introduction of part-of-speech leads to improvement of F-score in UWI by about 13.1 percents for HMMs and 2.31 percents for the lexicalized HMMs. As for the overall F-score in word segmentation, the improved number is 1.78 percents for HMMs and 0.23 percents for LHMMs.

In addition to the above experiment, we also conduct an open evaluation using the test-corpus for the track of PK-open in 2003 SIGHAN Bakeoff and compare our system with other public systems in the track. We have two reasons for the selection of this corpus: The first reason is that both the training data of this work and the SIGHAN-PK open test data are from Peking University. The second one is that some other resources such as the part-of-speech lexicon are used in our work, which does not satisfy the requirements for the closed tests at SIGHAN bakeoff. The results are presented in Table 3 and Table 4 .

Actually, Table 3 presents the results of the open test of the proposed methods on SIGHAN bakeoff data, which is parallel to Table 2. Comparing the data in the two tables, we find that they show the similar trends for different methods under discussion. We also notice that our system yields worse results in the open test. Our further error analysis shows that the drop of performance is caused by three main factors, namely the inconsistent segmentations between the training data and the open test data, the problem of data sparseness and the complicated cases that are beyond current methods.

Table 3. Experimental results on the corpus for SIGHAN-PK open test

\begin{tabular}{|l|c|c|c|}
\hline Methods & Recall(\%) & Precision(\%) & F-score(\%) \\
\hline HMMs & 93.31 & 91.09 & 92.19 \\
without & 97.20 & 92.04 & 94.55 \\
POS & 55.96 & 77.64 & 65.04 \\
\hline HMMs & 93.73 & 91.90 & 92.80 \\
With & 97.40 & 93.14 & 95.23 \\
POS & 58.43 & 75.68 & 65.95 \\
\hline LHMMs & 94.96 & 93.86 & 94.40 \\
without & 96.92 & 94.91 & 95.91 \\
POS & 76.10 & 82.63 & 79.23 \\
\hline LHMMs & $\mathbf{9 5 . 1 9}$ & $\mathbf{9 4 . 0 9}$ & 94.64 \\
With & $\mathbf{9 7 . 0 2}$ & $\mathbf{9 5 . 0 2}$ & $\mathbf{9 6 . 0 1}$ \\
POS & $\mathbf{7 7 . 5 8}$ & $\mathbf{8 4 . 2 4}$ & $\mathbf{8 0 . 7 7}$ \\
\hline
\end{tabular}

Table 4 presents the result of the comparison of our system with other open systems for the SIHAN-PK open test. As shown in Table 5, our system ranks the third in terms of the overall F-score in word-segmentation, which indicates in a sense that the proposed approach can yield results that are comparable to other state-of-the-arts approaches. Here, the system $\mathrm{S10}$ is developed based on the full sentence parsing technique and its results usually depend on a complicated fine tuning [10]; The system S01 is based on role-tagging technique, which need an additional role-tagged corpus for training [2]. In comparison with the two systems, our system is purely based on known word tagging and the lexicalized HMMs, and can be built efficiently on a manually segmented and part-of-speech corpus. This kind of corpus is now available for Chinese such as the PKU corpus and the CKIP corpus. At this point, our approach is more applicable.

Table 4. The comparison with the systems for the SIHAN-PK open test

\begin{tabular}{|l|c|c|c|c|c|}
\hline Systems & $\mathrm{R}_{\text {oov }}(\%)$ & $\mathrm{R}_{\text {iv }}(\%)$ & $\mathbf{R}(\%)$ & $\mathrm{P}(\%)$ & $\mathbf{F}(\%)$ \\
\hline S10 & 79.9 & 97.5 & 96.3 & 95.6 & 95.9 \\
\hline S01 & 74.3 & 98.0 & 96.3 & 94.3 & 95.3 \\
\hline S08 & 67.5 & 95.9 & 93.9 & 93.8 & 93.8 \\
\hline S04 & 71.2 & 94.9 & 93.3 & 94.2 & 93.7 \\
\hline S03 & 64.7 & 96.2 & 94.0 & 91.1 & 92.5 \\
\hline S11 & 50.3 & 93.4 & 90.5 & 86.9 & 88.6 \\
\hline $\begin{array}{l}\text { Our } \\
\text { system }\end{array}$ & $\mathbf{7 7 . 5 8}$ & $\mathbf{9 7 . 0 2}$ & $\mathbf{9 5 . 1 9}$ & $\mathbf{9 4 . 0 9}$ & $\mathbf{9 4 . 6 4}$ \\
\hline
\end{tabular}




\section{Conclusions}

In this paper, we have presented a lexicalized hidden Markov model approach to Chinese UWI. In this work, Chinese UWI is represented as a tagging task on a sequence of known words by introducing word-formation patterns. To do this work, a LHMM tagger is further developed on a manually part-of-speech tagged corpus to assign each known word in input an appropriate tag that indicates its patterns in forming a word and the part-of-speech of this formed word. In comparison with standard HMMs, the lexicalized HMMs can handle richer contextual information, both contextual words and tags for correct tagging of known words. In addition, part-of-speech tags are also introduced and further incorporated with the word-formation pattern tags. In this way, most Chinese unknown words can be resolved effectively. The experimental results on Peking University corpus indicate that the use of lexicalization technique and the introduction of POS are helpful to unknown word identification. The experiment on SIGHAN-PK open test data also shows that our system can achieve state-of-art performance. In practice, the proposed approach also provides a framework for part-of-speech tagging, in particular for unknown word tagging. In future work, we hope to apply it in Chinese part-of-speech tagging and other NLP applications such as named entity recognition.

\section{Acknowledgements}

We would like to thank the Institute of Computational Linguistics, Peking University for providing part-of-speech tagset, lexicon and corpus for our experiments.

\section{References}

[1] Andi Wu, and Zixin Jiang, "Statistically-enhanced new word identification in a rule-based Chinese system", Proceedings of the Second Chinese Language Processing Workshop, Hong Kong, pp. 46-51, 2000.

[2] Hua-Ping Zhang, Qun Liu, Hao Zhang, and Xue-Qi Cheng, "Automatic recognition of Chinese unknown words based on roles tagging", Proceedings of The First SIGHAN Workshop on Chinese Language Processing, Taiwan, pp.71-77, July 2002.

[3] Nianwen Xue, "Chinese word segmentation as character tagging", Computational Linguistics and Chinese Language Processing, Vol 8, No.1, pp.29-48, Feb. 2003.

[4] Guohong Fu, and Kang-Kwong Luke, "Chinese unknown word identification using class-based LM", Proceedings of The First International Joint Conference on Natural Language Processing (IJCNLP-04), Hainan Island, China, pp.262-269, March 2004.

[5] Sang-Zoo Lee, Jun-ichi Tsujii, and Hae-Chang Rim, "Lexicalized hidden Markov models for part-of-speech tagging", Proceeding of The $18^{\text {th }}$ Conference on Computational Linguistics (COLING 2000), Saarbruken, Germany, pp. 481-487, July 2000.

[6] Antonio Molina, and Perran Pla, "Shallow parsing using specialized HMMs", Journal of Machine Learning Research, Vol. 2, pp.595-613, March 2002.

[7] Guohong Fu, and Kang-Kwong Luke, "Integrated approaches to prosodic word prediction for Chinese TTS", Proceedings of the 2003 IEEE International Conference on Natural Language Processing and Knowledge Engineering (NLP-KE03), Beijing, China, pp. 413-418, Oct. 2003.

[8] Shiwen Yu, Huiming Duan, Suefeng Zhu, Bin Swen, and Baobao Chang, "Specification for corpus processing at Peking University: Word segmentation, POS tagging and phonetic notation", Journal of Chinese Language and Computing, Vol.13, No.2, pp. 121-158, 2003.

[9] Richard Sproat, and Thomas Emerson, "The first international Chinese word segmentation bakeoff", Proceedings of the Second SIGHAN Workshop on Chinese Language Processing, Sapporo, Japan, pp.133-143, July 2003.

[10] Andi Wu, "Chinese word segmentation in MSR-NLP", Proceedings of the Second SIGHAN' Workshop on Chinese Language Processing, Sapporo, Japan, pp. 172-175, July 2003. 